Lena Lehmann / Katharina Leimbach

\title{
„Schuster bleib bei deinem Leisten!“ - Unterschiede zwischen qualitativen Interviews und polizeilichen Vernehmungen
}

\section{Abstract}

Im Themenfeld der Sicherheitsforschung sind Analysen und das Voranbringen von Erkenntnissen ohne eine Kooperation zwischen Wissenschaft und Sicherheitsakteur*innen nicht möglich. Sicherheitsakteur*innen und Wissenschaftler*innen verfolgen unterschiedliche Handlungslogiken und verfügen somit über verschiedene Zugänge zu Themenbereichen, Daten und Personengruppen. Diese unterschiedlichen Zugänge sind für eine Analyse, Problematisierung und das Erkenntnisinteresse von großer Bedeutung. Ein Beispiel für eine kooperative und vertrauensvolle Zusammenarbeit stellt die Radikalisierungsforschung dar. Am Beispiel des qualitativen Interviews der sozialwissenschaftlichen Forschung und der polizeilichen Vernehmung werden die Unterschiede und Gemeinsamkeiten dargelegt. Dabei wird verdeutlicht, worin die Gefahr besteht, wenn die Freiheit von Wissenschaftlichkeit konterkariert wird und warum es $\mathrm{u}$. a. unabdingbar ist, dass der Artikel 5 des Grundgesetzes in seiner Form von allen Akteur*innen gewahrt wird.

Schlagwörter: Qualitative Kriminologie; qualitative Interviews; sozialwissenschaftliche Sicherheitsforschung; polizeiliche Vernehmung; Radikalisierungsforschung

\section{Differences between qualitative interviews and police interrogation}

\section{Abstract}

To generate new knowledge in security research it is necessary to have a cooperation between scientists and actors in security matters. Since both parties follow different interests, their approaches towards issues, data and people differs from each other. To examine those approaches it is important to further understand their analyses, problematization and knowledge interests. One example for a trusting cooperation is research regarding radicalization. Using qualitative interviews and police interrogation as examples this article demonstrates its differences and similarities. It is shown that in order to protect the freedom of science everyone involved has to grant article 5 of Germanys constitution. 
Keywords: Qualitative criminology; qualitative interviews; security research; radicalization research; police interrogation

\section{A. Einfübrung}

Das Erkenntnisinteresse von Sicherheitsorganen und Wissenschaftler\%innen kann auf vielfältige Weise differieren ${ }^{1}$. Dies liegt großteils in der Natur der Sache. Das bedeutet keineswegs, dass eine Zusammenarbeit im wissenschaftlichen Sinne nicht stattfindet oder unerwünscht ist. Ganz im Gegenteil, denn im Themenfeld von Sicherheitsforschung ist ohne eine Kooperation zwischen Wissenschaft und Sicherheitsakteur\%innen kein Erkenntnisgewinn möglich. Dies liegt darin begründet, dass Sicherheitsakteur"innen und Wissenschaftler*innen verschiedene Zugänge zu Themenbereichen, Daten und Personengruppen haben. Diese unterschiedlichen Zugänge sind für eine Analyse, Problematisierung und das Erkenntnisinteresse von großer Bedeutung. Ein Beispiel für eine kooperative und vertrauensvolle Zusammenarbeit stellt die Radikalisierungsforschung dar. Wissenschaftliche Akteur\%innen verfolgen unter der Prämisse der Freiheit der Wissenschaft (Art. 5 GG) einen breiten Ansatz, um den Themenfeldern Radikalisierung und Extremismus näher zu kommen. Hierbei geht es u. a. häufig darum, zu erforschen, wie, wann, warum und in welcher Form sich Radikalisierung vollzieht (z. B. Prädiktoren), aber auch, inwiefern hier Theorien generiert werden können, um verlässliche Aussagen zu treffen. Diese Erkenntnisse dienen vornehmlich dazu, Präventionsangebote zu entwickeln, zu erweitern oder ganz neu aufzustellen und haben damit einen starken Anwendungsbezug ${ }^{2}$. Gleichzeitig können die gewonnenen Erkenntnisse auch für Sicherheitsakteur*innen in ihrer Arbeit von hoher Bedeutung sein. Dabei stützt sich die Wissenschaft auf verschiedene wissenschaftliche Methoden, um zu einer Erkenntnisgenerierung zu gelangen. Häufig werden im Bereich der Radikalisierungsforschung Personen u. a. mit Hilfe von qualitativen Erhebungsmethoden (z. B. im Rahmen von qualitativen Interviews) befragt. Der Zugang zu diesen Personen entsteht nicht in einem luftleeren Raum, sondern wird vielmehr von verschiedenen Sicherheitsakteur*innen unterstützt bzw. der Zugang dazu ermöglicht. Um ihrem Auftrag nachzukommen, führen Sicherheitsakteur"innen in ihrem arbeitsrechtlichen Rahmen ebenfalls eine Art von Interview durch. Dabei handelt es sich vielmehr um Befragungen und Vernehmungen, die, wie auch bei der qualitativen Forschung, verschiedenen Kriterien und Ansprüchen unterliegen. Diese differieren aber in starkem Maße von denen der Wissenschaft. Dies hängt mit den verschiedenen Erkenntnisinteressen und den rechtlichen Rahmenbedingungen zusammen. Warum kommt es dennoch zu Irritatio-

1 Hier sei allein der Diskurs zum Themengebiet der Polizeiforschung benannt. So stellt sich hier die Frage, ob für oder über die Polizei geforscht wird (vgl. Initiative Polizei und Wissenschaft 2017).

2 Während anwendungsorientierte Forschung das Ziel einer praktischen Verwertbarkeit von Forschungsergebnissen verfolgt, fungiert Grundlagenforschung auf einem höheren Abstraktionsniveau und lässt sich seltener in fachpraktische oder politische Handlungsempfehlungen übersetzen. 
nen? Dies liegt nicht nur an dem gemeinsam verwendeten, aber völlig unterschiedlich ausgestalteten Begriff der „Befragung“, sondern auch an einer zunehmenden Dominanz von Sicherheitsinteressen und einer Verwobenheit des Wissenschafts- und Sicherheitssystems ${ }^{3}$. Der wissenschaftliche Auftrag, Erkenntnisse über Radikalisierung zu generieren, die sich hinterher politisch und fachpraktisch umsetzen lassen, überschneidet sich mit jenem von Sicherheitsbehörden dann, wenn Radikalisierte detaillierte Auskünfte über ihre Motivationen und ihre biographischen Zusammenhänge geben. Hier werden mitunter Informationen geliefert, die sowohl wissenschaftlichem Erkenntnisinteresse dienen als auch sachdienliche Hinweise zur Einhaltung der sozialen Ordnung oder zur Verhinderung von Straftaten liefern können.

Der folgende Beitrag widmet sich den Unterschieden und Gemeinsamkeiten von qualitativen Interviews und polizeilichen Vernehmungen und verdeutlicht, worin die Besonderheiten in den jeweiligen Befragungsmethoden liegen. Anschließend wird die Notwendigkeit einer Trennung aus soziologischer Perspektive umrissen. Dabei soll deutlich werden, dass es eine von Sicherheitsinteressen unabhängige Forschung geben muss. Dies ist insofern von Bedeutung, als dass auch staatliche Praktiken sich einer kritischen Würdigung von Wissenschaft unterziehen müssen. Gerade durch Wissenschaft können Entwicklungen, Implikationen und Neuerungen geschaffen werden, um auch gesamtgesellschaftliche Prozesse zu berücksichtigen.

Zunächst wird auf das methodische Vorgehen der qualitativen Forschung eingegangen, um daran anschließend die polizeiliche Vernehmung und ein mögliches Vorgehen bei Befragungen zu verdeutlichen. Hierbei können die beiden Themenbereiche nur in ihren wesentlichen Grundzügen skizziert werden.

\section{B. Qualitative Sozialforschung und Interviews als Methode der Datenerhebung}

Wissenschaft verfolgt das Ziel, neue Erkenntnisse zu generieren. Der Erkenntnisprozess als wissenschaftliche Arbeitsweise erfolgt dabei regelgeleitet und transparent. Die regelgeleitete bzw. methodisch angeleitete Untersuchung von sozialen Wirklichkeiten ist der Selbstzweck empirischer Wissenschaft. Hierbei wird zwischen quantitativen (standardisierten) und qualitativen (interpretativen) Verfahren unterschieden ${ }^{4}$. Im Fokus qualitativ-angeleiteter Forschungsfragen steht das „Verstehen“ von sozialen Prozessen und von sozialem Handeln ${ }^{5}$. Qualitative Methoden sind „kontrollierte Methoden des Fremdverstehens"6. Das Portfolio qualitativer Methoden beinhaltet Erhebungsmethoden in Form der Datengenerierung sowie Auswertungsmethoden und die damit verbundene Datenanalyse. Eine Variante, um qualitative Daten regelgeleitet zu erheben, sind qualitative Interviews. Diese können nach dem Grad ihrer Offenheit bzw. Geschlossenheit unterschieden werden: Von narrativen Interviews bis hin zu leit-

3 Jukschat/Leimbach Behemoth-Journal 12/2 (2019), 11, 13.

4 Diekmann 2011, 33 ff.; Strübing 2013, 3 ff.

5 Bohnsack 2014, 22 ff.; Kleemann et al. 2013, 13; Przyborski/Woblrab-Sabr 2014, 16.

6 Schütze et al. 1973.

NK 32. Jg. $3 / 2020$ 
fadengestützten Interviews ${ }^{7}$. Alle Interviews haben gemein, dass sie aufgenommen, transkribiert, anonymisiert und anschließend ausgewertet werden.

Grundlegend ist die Annahme, dass Gesellschaft symbolisch interaktiv ${ }^{8}$ ist und kommunikativ hervorgebracht wird 9 . Je nachdem, ob die Methode des Interviews eingesetzt wird, um das „Was wird kommuniziert“ oder um das „Wie wird etwas kommuniziert" nachzuvollziehen, sollen Konstruktionen 1. Ordnung oder 2. Ordnung erkenntnistheoretisch geleitet expliziert werden ${ }^{10}$.

Mit qualitativen Interviews, also dem freiwilligen Sprechen mit Personen, die nach bestimmten Kriterien ausgewählt wurden ${ }^{11}$, werden Informationen eingeholt, die sich am Forschungsinteresse auszurichten haben und in der Regel soziale Strukturen, Prozesse, Entscheidungen und Praktiken fokussieren. Da hierbei nicht die interviewte Person selbst im Vordergrund steht, sondern das, was sie repräsentiert, finden qualitative Interviews ausschließlich unter der Prämisse von Anonymität statt.

\section{Gütekriterien und Ziele qualitativer Sozialforschung}

Der Erkenntnisgewinn ist das Ziel wissenschaftlicher Arbeit, der Weg zum Erkenntnisgewinn ist regelgeleitet. Das regelgeleitete oder methodische Vorgehen muss wissenschaftlichen Standards entsprechen und wird an bestimmten Kriterien bemessen. So werden auch in der qualitativen Sozialforschung Gütekriterien zu Grunde gelegt. Nur durch Einhaltung dieser Kriterien werden Annahmen zu wissenschaftlichen Aussagen. Zudem können anhand der Kriterien wissenschaftliche Studien eingeordnet und kritisch geprüft werden. In der qualitativen Sozialforschung können neben der Reliabilität (Zuverlässigkeit), Validität (Gültigkeit) und Objektivität (im Sinne intersubjektiver Nachvollziehbarkeit) ${ }^{12}$ noch weitere Kriterien identifiziert werden, die im Folgenden kommentiert aufgelistet sind:

- Offenheit: Der Grad der Offenheit kann sich von Methode zu Methode unterschei$\operatorname{den}^{13}$. Zur Erforschung bisher unbekannter oder minderbekannter Phänomene ist methodisch kontrollierte Offenheit gegenüber dem Erkenntnisprozess und den Ergebnissen die notwendige Bedingung für einen explorativen Forschungsprozess ${ }^{14}$.

7 Helfferich 2011; Heiser 2018, $51 \mathrm{ff}$.

8 Der symbolische Interaktionismus ist eine Forschungstradition, die auch als „Chicago School" bezeichnet wird und nicht nur für die Entwicklung qualitativer Methoden grundlegend war, sondern auch für die interaktionistische Strömung in der Kriminologie (Miller et al. 2015, $3 \mathrm{ff}$.).

9 Przyborski/Woblrab-Sabr 2014, 79.

10 Kleemann et al. 2013, 19 ff.; Przyborski/Woblrab-Sabr 2014, $13 \mathrm{ff}$.

11 Przyborski/Woblrab-Sabr 2014, $177 \mathrm{ff}$.

12 Dies. 2014, 28.

13 Rosenthal 2015, 14.

14 Kleemann et al. 2013, 19. 
- Gegenstandsangemessenheit: Forschungsfrage, Forschungsmethodik und Forschungsgegenstand müssen aneinander ausgerichtet und zueinander passfähig $\operatorname{sein}^{15}$.

- Intersubjektive Nachvollziehbarkeit: Jegliche Entscheidungen, die den Forschungsprozess und insbesondere die Datenanalyse betreffen, müssen für Dritte nachvollziehbar $\operatorname{sein}^{16}$.

- Reflexivität: Es gilt, ein ständiges und transparentes Bewusstsein über die Entstehung von Phänomenen durch reziproke Verweisungszusammenhänge zu haben und dieses nachvollziehbar zu reflektieren. So ist etwa das wechselseitige Formen von Forschungsfrage und Forschungsgegenstand ebenso wie die eigene Rolle im Feld als Wissenschaftler*in zu berücksichtigen ${ }^{17}$.

- Empirische Verankerung/Sättigung: Eine induktive und abduktive Forschungslogik sind einzuhalten. Finale Theoretisierungen gehen aus den Daten bzw. der Empirie hervor $^{18}$.

- Kohärenz und Limitation: Daten und Interpretationen müssen verhältnismäßig zueinander sein. Interpretationen müssen in der Empirie verankert und angemessen sein. Generalisierungen sind limitiert und müssen in ihrem Niveau bestimmt wer$\operatorname{den}^{19}$.

- Originalität und Relevanz: Wenn das Ziel der Wissenschaft der Erkenntnisgewinn ist, dann müssen empirische Studien ihre Relevanz und Originalität belegen ${ }^{20}$.

Qualitative Verfahren sind ein Weg, aus geistes- und sozialwissenschaftlich-verorteter Forschung wissenschaftliche Erkenntnisse zu generieren. Um wissenschaftlichen Ansprüchen gerecht zu werden, müssen die dargelegten Kriterien in ihrer Vollständigkeit anerkannt und eingehalten werden. Qualitative Interviews sind eine Variante, um wissenschaftliche Daten zu erheben, die so offen wie möglich und geschlossen (in Bezug auf die Fragestellung und das Forschungsinteresse) wie nötig abzulaufen haben. Die Datenerhebung darf dabei allein einem wissenschaftlichen Erkenntnisinteresse gelten. Auch die Datenauswertung muss in Bezug auf das Forschungsinteresse und entlang der methodischen Standards erfolgen. Demnach wird mit qualitativen Interviews Wissen über Personen eingeholt, das jedoch allein den Sinn und Zweck des wissenschaftlichen Erkenntnisinteresses fokussiert.

\section{Vernehmungen}

Vernehmungen werden von unterschiedlichen Amtspersonen durchgeführt und dienen zur Aufklärung eines Sachverhalts im Strafprozessrecht. Das „Ziel von einer Verneh-

15 Strübing et al. ZfS 2018, 83, 86.

16 Rosenthal 2015, 18.

17 Strübing 2013, $125 \mathrm{ff}$.

18 Kleemann et al. 2013, 22 ff.; Strübing et al. ZfS 2018, 83, 88.

19 Przyborski/Woblrab-Sabr 2014, $363 \mathrm{ff}$.

20 Strübing et al. ZfS 2018, 83, 95.

NK 32. Jg. $3 / 2020$ 
mung ist das Herausfinden der objektiven Wahrheit, die Erforschung des Sachverhaltes und die Rekonstruktion des Tatherganges“21. Darüber hinaus sollen relevante gerichtsverwertbare und beweisrelevante Informationen gewonnen werden, die auch dazu dienen, Beschuldigte zu be- oder entlasten ${ }^{22}$. Dabei müssen rechtliche Regeln eingehalten werden.

So finden Vernehmungen durch Richter*innen, Staatsanwält*innen oder auch Polizeivollzugsbeamt*innen statt. Diese Institutionen sozialer Kontrolle sind angehalten, die Ermittlungen bei Normabweichungen zu führen und letztlich die Schuld- und Straffrage für die zuvor festgestellte Normabweichungen zu klären ${ }^{23}$. Entscheidend dabei ist, dass Vernehmungen eine auf Rechtsvorschriften beruhende Methode darstellen. Die Auskunftsperson, die einem*r Richter*in, Staatsanwält*in oder der Polizeivollzugsbeamt”in gegenübersteht, wird dabei in die Kategorie „Zeug*in“ oder „Beschuldigte ${ }^{*}$ " eingeordnet. Dabei kommen verschiedene Techniken und Taktiken zum Einsatz, um letztlich eine Tatsachenfeststellung zu erlangen. Je nach Berufsgruppe (z. B. Richter\%in, Staatsanwält $*$ in) bestehen unterschiedliche Erkenntnisinteressen in einem Verfahren. Dabei existieren verschiedene "Grundregeln“ der Vernehmungslehre, wie z. B. das Stellen von: Filterfragen, möglichst offenen W-Fragen (Wer? Wo? Wie? Etc.), Leerfragen, Anstoßfragen, Situationsfragen. So kann festgehalten werden: "Jede Vernehmungs- und Fragetechnik, insbesondere im forensischen Bereich, ist immer zugleich Vernehmungs-TAKTIK!“24. Im Wesentlichen geht es darum, zu identifizieren, was Lüge und Wahrheit ist. Dabei unterscheidet Shibles (2000) drei Kategorien: 1) Was wahr ist; 2) Was eine Person subjektiv erinnert/glaubt; 3) Was eine Person explizit sagt. Letztere Kategorie umfasst die Lüge, „wenn eine Person etwas anderes sagt, als sie subjektiv glaubt" ${ }^{25}$.

Eine Schwierigkeit, die dabei besteht, ist die Prüfung der Glaubhaftigkeit der Aussagen. Die Glaubhaftigkeit von Aussagen ist eng verzahnt mit der Vernehmungsmethode. Im Vordergrund des Vernehmungsziels steht dabei die Wahrheit ${ }^{26}$.

\section{E. Polizeiliche Vernebmungen}

Als eine Methode, um den Sachverhalt zu erhellen, kann bspw. das narrative Interview gewählt werden, welches auch in der qualitativen Forschung zum Einsatz kommt. Ähnlich wie in der Forschung ist es bei dieser Anwendung der Methode von Bedeutung, dass eine gewisse Offenheit (z. B. durch Freundlichkeit und einen ruhigen Ton) von der fragenden Person ausgeht. Auch wird, wie in der qualitativen Forschung, die aussagende Person eingangs nicht direkt zum Hauptthema befragt, sondern ein Stimulus gesetzt. Das Ziel in diesem Fall ist es, „(...) dass er seine vorgefertigte Geschichte

21 Hermanutz/Litzcke 2009, 18.

22 Dies. 2009, 19.

23 Wendler/Hoffmann, 2015, 2.

24 Dies. 2015, 8.

25 Hermanutz/Litzcke 2009, 20.

26 Dies. 2009, 124. 
nicht an den Mann bringen kann und damit verunsichert wird“27. Hierin liegt bereits der erste Unterschied zur wissenschaftlichen qualitativen Interviewführung. Die Personen in der qualitativen Forschung sollen eben nicht verunsichert werden, sondern ganz im Gegenteil, ihnen soll ein Raum der Sicherheit und Unvoreingenommenheit als auch Glaubhaftigkeit in der Aussage eröffnet werden, um einen Zugang zu erhalten. Bei der polizeilichen Vernehmung dient dies eher dazu, das Verhalten der Person während der Befragung zu verschiedenen Zeitpunkten zu vergleichen. Das „Nicht-Unterbrechen" während der Erzählung gilt hier ebenso, wie auch in der qualitativen Forschung. Wenn nach dem Bericht noch weitere Aspekte bestehen, die Nachfragen erfordern, dann können diese gezielt gestellt werden. Dies ist ebenso in der qualitativen Forschung zu finden. In einem weiteren Schritt schließt das Verhör an, welches Bezugsfragen an die Aussageperson richtet und auch durch weitere konkrete und gezieltere Fragen Widersprüchlichkeiten aufdecken bzw. klären soll28. Diese Vorgehensweise dient dazu, u. a. noch weitere Informationen zum Sachverhalt zu erlangen.

Neben dem methodischen Vorgehen bei der Vernehmung stellen die Kommunikation und das Verhältnis zwischen zu vernehmender und vernehmender Person einen weiteren Aspekt dar, der den Unterschied zwischen Vernehmung und sozialwissenschaftlicher Befragung verdeutlicht. So besteht ein Ungleichgewicht in der Kommunikation zwischen fragender und befragter Person. Gerson (2016) beschreibt in diesem Zusammenhang, dass das Strukturprinzip der Vernehmung auf Ungleichheit ausgelegt is $^{29}$. So ist die Kommunikation in der Vernehmung kein „üblicher Kommunikationsprozess“, sondern stellt „in gewisser Weise einen einseitig staatlich veranlassten Zwangskommunikationsprozess dar" 30 . Dabei wird auch deutlich, dass es bei der Vernehmung nicht nur um den reinen Austausch von Informationen geht, sondern vielmehr ein Machtgefälle zwischen der Aussageperson und der fragenden Person besteht. Gerson (2016) bezeichnet die Vernehmung als eine „intendierte Demonstration von Macht und Einfluss“"31. Insbesondere die Tatsache, dass die befragende Person als Vertreter\%in des Staates auftritt, weist auf diese Differenzen hin. Hier wird der Unterschied zwischen polizeilicher Vernehmung mit dem Ziel, einseitig nach „Wahrheiten“ zu forschen und den sozialwissenschaftlichen Methoden mit dem Ziel bspw. Lebenswelten zu ergründen, deutlich. Während es sich bei Vernehmungen durch die oben aufgeführten differenten Rollen („Über- und Unterordnungsverhältnis“) eher um eine Zwangskommunikation handelt, basiert die sozialwissenschaftliche Erhebung auf Freiwilligkeit des Gegenübers, die Bereitschaft und Offenheit aus seiner Perspektive zu berichten. Gleichzeitig soll die Kommunikation zwischen forschender und interviewter Person auf gleicher Ebene („Augenhöhe“) stattfinden. Darüber hinaus wird in dem Ort der Befragung ebenfalls der Unterschied deutlich. Bei Vernehmungen findet dies vornehmlich in polizeilichen Räumen statt, d. h. die zu befragende Person befindet

27 Dies. 2009, 125.

28 Dies. 2009, 127.

29 Gerson 2016, 497.

30 Kroll SIAK-Journal 2014, 17, 18.

31 Gerson 2016, 497.

NK 32. Jg. $3 / 2020$ 
sich in fremder Umgebung. Hingegen findet das Interview in der wissenschaftlichen Praxis häufig an einem von der interviewten Person gewählten Ort statt. Dies dient vornehmlich dazu, dass sich die zu befragende Person im geschützten Raum „sicher“ fühlt und somit die Wahrscheinlichkeit höher ist, dass sie sich auf das Gespräch und die Thematik gänzlich einlässt und öffnet. Während bei Vernehmungen die Thematik von falschen Geständnissen eine Rolle spielt ${ }^{32}$, ist in der sozialwissenschaftlichen Interviewführung die Kategorisierung in „wahr" oder „falsch" von Aussagen nicht existent, da hier nicht nach der Wahrheit geforscht wird, sondern nach Perspektiven auf Phänomene resp. die Subjektivierung sozialer Phänomene. Es lassen sich weitere Aspekte identifizieren, an denen sich die Unterschiede zwischen Vernehmungen und sozialwissenschaftlicher Datenerhebung verdeutlichen. Die wesentlichen Zielrichtungen der unterschiedlichen Vorgehensweisen und Intentionen dieser Bereiche sollten aber auf diese Weise deutlich geworden sein.

\section{F. Warum Äpfel mit Birnen vergleichen? Ein kritisches Fazit}

Die skizzierten Ausführungen sollen die unterschiedlichen Gemeinsamkeiten und Praktiken verdeutlichen, aber insbesondere die unterschiedlichen Handlungsziele als wichtigstes Unterscheidungsmerkmal konstatieren. Während die Sozialwissenschaften auch immer die gesamtgesellschaftliche Perspektive betrachten und somit der wissenschaftliche Erkenntnisgewinn über eine individuelle Wahrheit hinausgeht, folgt die Vernehmung bzw. das Verhör einer anderen Logik. Hier geht es um die Identifikation der „objektiven Wahrheit“ und darum, Sachverhalte aufzuklären, sodass diese einer kritischen Prüfung vor Gericht standhalten. Warum also wissenschaftliche Interviews und polizeiliche Vernehmungen darstellen und voneinander abgrenzen? Unsere dreijährige Feldforschung zum Thema Radikalisierung hat gezeigt, dass sich, obwohl die Ziele wissenschaftlichen und sicherheitsbehördlichen Arbeitens klar zu unterscheiden sind, beide in der Praxis vermengen. Der Zugang zu bestimmten Personengruppen kann aufgrund verschiedener Aspekte (z. B. Haftstrafe) nur über sicherheitsbehördliche Wege erfolgen. Dies kann etwa bedeuten, dass der Zugang zu bestimmten Personen für die Durchführung von Interviews verwehrt wird oder dass, wie in der Protestnote benannt, es zu einer Beschlagnahme von wissenschaftlichem Interviewmaterial (ohne Abwägung der Einschränkung der Wissenschaftsfreiheit) kommt. Ersteres führt zu einer Verzerrung der Befragtenpopulation und hat damit direkte Auswirkungen auf die Forschungsergebnisse. Zweites kann zukünftige Forschungen und die Bereitwilligkeit von Personen, an Interviews teilzunehmen, maßgeblich beeinflussen und erschweren. Dies liegt darin begründet, dass die Gewährung von Anonymisierung und NichtWeitergabe an Dritte außerhalb von Forschungszwecken durch die Wissenschaftler"innen nicht uneingeschränkt zugesichert werden kann, da die Befürchtung besteht, dass hier von Sicherheitsbehörden eingegriffen wird. Dies führt dazu, dass das Vertrauen potentieller Interviewpartner*innen nachhaltig geschädigt wird und der Zugang zu

32 Kroll SIAK-Journal 2014, 17, $21 \mathrm{f}$.

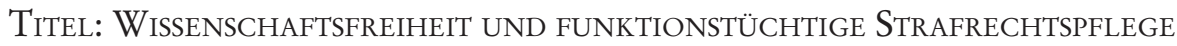


diesen nicht länger möglich ist. Nicht nur, dass es schwieriger wird, Personen für Interviews zu gewinnen, auch muss sich die Frage für uns als Wissenschaftler*innen stellen, inwieweit wir Forschungen überhaupt realisieren können/wollen, wenn die Durchführung von Forschungsprojekten in ihren Auswirkungen nicht mehr kontrollierbar ist bzw. die Wissenschaftsfreiheit beschnitten wird. Gerade in einem hochsensiblen Bereich der Sicherheitsforschung erscheint es von besonderer Bedeutung, sich die verschiedenen Perspektiven mit ihren Logiken der Sicherheitsakteur"innen aber auch der Wissenschaflter*innen zu verdeutlichen. ${ }^{33}$ Sicherheit ist ein normatives und politisches Konzept, in dem sich Herrschaftswissen und Machtstrukturen abbilden. Sozialwissenschaftliche Sicherheitsforschung reproduziert und legitimiert diese Strukturen allein durch ihre Existenz ${ }^{34}$. Reflexives Forschen heißt auch, sich dieser Eingebundenheit bewusst zu sein, und macht sie in unserer Meinung nicht per se unmöglich. Die Praktik des Forschens wird erst dann unmöglich, wenn wissenschaftliche Praktiken intendiert instrumentalisiert werden und das eine für das andere in Anspruch genommen wird. In der Kriminologie, als meist anwendungsorientierte Bezugswissenschaft, kondensiert sich die Problematik einer Trennung zwischen wissenschaftlichen und sicherheitsbehördlichen Interessen. Je stärker der Forschungsgegenstand gesellschaftlich problematisiert wird und unter der Kategorie „(inter-)nationales Sicherheitsrisiko“ läuft, desto schwieriger wird unabhängige, ergebnisoffene Wissenschaft ${ }^{35}$. Die Beschneidung von Wissenschaftsfreiheit hat aber nicht nur direkte Konsequenzen für das Forschungsfeld und akademische Praktiken, sondern ist auch eine Gefahr für eine liberale Demokratie. Die Auswirkungen der Verschärfung von Terrorabwehrgesetzen und ihre Auswirkungen auf gesellschaftliche und demokratische Freiheiten sind bereits vielfach besprochen worden ${ }^{36}$. Die Durchführung unserer Forschungen im Themengebiet Radikalisierung und Extremismus haben gezeigt wie wichtig es ist, die Unterschiedlichkeit wissenschaftlicher und sicherheitsbehördlicher Praktiken zu konstatieren und damit die Forderung zu verbinden: „Schuster bleib bei deinem Leisten!“. Nur so werden Wissenschaftler*innen weiter in der Lage sein, vom Grundgesetz geschützte und ergebnisoffene Erkenntnisprozesse über gesellschaftliche Phänomene (unabhängig von ihrer Sicherheitsrelevanz) zu produzieren und zur Erhaltung demokratischer Grundprinzipien beizutragen ${ }^{37}$. Daher ist es u. a. unabdingbar, dass der Artikel 5 des Grundgesetzes in seiner Form von allen Akteur*innen gewahrt wird.

33 Initiative Polizei und Wissenschaft 2017, 258.

34 Webrheim KrimJ 2018, 211, 218.

35 Aktuelle Entwicklungen und Debatten rund um Drittmittelförderungen von Forschungsprojekten, die auf diese Weise angehalten sind, unmittelbaren Praxisnutzen zu generieren, sind ein weiterer wichtiger Aspekt bei der Versicherheitlichung partikularer Gesellschaftssysteme, was an dieser Stelle jedoch nicht weiter behandelt werden kann (siehe z.B. Wehrbeim 2013).

36 Vgl. Puschke/Singelnstein 2018; Masing JZ 2011), 753 ff.; Jasch KrimJ 2007, 203 ff.

37 Siehe hierzu auch die von der DFG-finanzierten Thesen der Wissenschaftsfreiheit: https:// wissenschaftsfreiheit.de/abschlussmemorandum-der-kampagne/ (Letzter Zugriff: 23.5.2020).

NK 32. Jg. $3 / 2020$ 


\section{Literatur}

Bohnsack (2014) Rekonstruktive Sozialforschung. 9. Aufl.

Diekmann (2011) Empirische Sozialforschung. Grundlagen, Methoden, Anwendungen. 5. Aufl.

Gerson (2016) Das Recht auf Beschuldigung: strafprozessuale Verfahrensbalance durch kommunikative Autonomie

Heiser (2018) Meilensteine der qualitativen Sozialforschung. Eine Einführung entlang klassischer Studien

Helfferich (2011) Die Qualität qualitativer Daten. Manual für die Durchführung qualitativer Interviews. 4. Aufl.

Hermanutz/Litzcke (2009) Vernehmung in Theorie und Praxis. Wahrheit - Irrtum Lüge. 2. Aufl.

Initiative Polizei und Wissenschaft (2018) Polizei und Wissenschaft - eine kritische Standortbestimmung. In: Lange /Wendekamm (Hrsg.): Die Verwaltung der Sicherheit. Theorie und Praxis der öffentlichen Sicherheitsverwaltung, 257

Jasch Strafrecht im Dilemma zwischen Sicherheit und Freiheit, in: KrimJ 2007, 203-213 Jukschat/Leimbach Radikalisierung als hegemoniales Paradigma. Eine empiriebasierte kritische Bestandsaufnahme, in: Behemoth Journal 12/2 (2019), 11-23

Kleemann/Kräbnke/Matuschek (2013) Grundlagen interpretativer Sozialforschung, in: Kleemann/Krähnke/Matuschek (Hrsg.), Interpretative Sozialforschung, 13

Kroll Wahre und falsche Geständnisse in Vernehmungen, in: SIAK-Journal - Zeitschrift für Polizeiwissenschaft und polizeiliche Praxis 2014/2, 17-32 (Online abrufbar: http://dx.doi.org/10.7396/2014_2_B)

Masing Die Ambivalenz von Freiheit und Sicherheit, in: JZ 66 (2011), 753-758

Miller/Copes/Hochstetler (2015) The History and Evolution of Qualitative Criminology, in: Copes/Miller (Hrsg.), The Routledge Handbook of Qualitative Criminology, 3-21.

Przyborski/Woblrab-Sabr, (2014) Qualitative Sozialforschung. Ein Arbeitsbuch, 4. Aufl.

Puschke/Singelnstein (2018) Der Staat und die Sicherheitsgesellschaft.

Rosenthal (2015) Interpretative Sozialforschung: eine Einführung, 5. Aufl.

Scbütze/Meinefeld/Springer/Weymann (1973) Grundlagentheoretische Voraussetzungen methodisch kontrollierten Fremdverstehens, in: Arbeitsgruppe Bielefelder Soziologen, 433-495 
Shibles (2000) Lügen und lügen lassen: eine kritische Analyse des Lügens

Strübing (2013) Qualitative Sozialforschung. Eine komprimierte Einführung für Studierende

Strübing/Hirschaner/Ayaß/Krähnke/Scheffer Gütekriterien qualitativer Sozialforschung. Ein Diskussionsanstoß, in: ZfS 2018, 83-100

Webrheim (2018) Kritik der Versicherheitlichung: Thesen zur (sozialwissenschaftlichen) Sicherheitsforschung, in: KrimJ 2018, 211-221 (Online abrufbar: https:// www.beltz.de/fileadmin/beltz/newsletter/pdf/kj18_3_wehrheim.pdf.)

Wendler/Hoffmann (2015) Technik und Taktik der Befragung: Prüfung von Angaben, Gespräche, Interviews und Vernehmungen zielorientiert vorbereiten und führen, Urteile richtig begründen, Fehler in Urteilen aufdecken, Lüge und Irrtum erkennen, 2. Aufl.

\section{Kontakt}

Dr. Lena Lehmann

Kriminologisches Forschungsinstitut Niedersachsen e.V.

Lützerodestraße 9

30161 Hannover

Lena.Lehmann@kfn.de

Katharina Leimbach

Leibniz-Institut Hessische Stiftung Friedens- und Konfliktforschung

Baseler Straße 27-3

60329 Frankfurt am Main

leimbach@hsfk.de 